\title{
Inference of power loss in spherical joints of the 6RSS parallel mechanism
}

\author{
Lucian Milica ${ }^{1, *}$ and Gabriel Andrei ${ }^{1}$ \\ 1"Dunarea de Jos" University, Department Mechanical Engineering, 800201 Galati, Romania
}

\begin{abstract}
This paper presents an analysis of the power losses due to the friction between the components of the spherical joints, belonging to a 6 RSS parallel mechanism. The determination of power losses in spherical joints has been done with an application where the characteristic point follows a closed space curve. The trajectory of the point on the convex semicuple in contact with the concave semicuple it has been determined by using facilities of the CATIA software.
\end{abstract}

\section{Introduction}

The paper presents a method for determining the power losses for a parallel mechanism by friction between the components of the spherical joints that form its six kinematic chains. Spherical joints are a special category of joints with the particularity that the contact surfaces are spherical, with or without lubricant between them.

The importance of spherical joints has led many researchers to carry out numerous studies to determine their influence on the dynamics of mechanical systems [1-4]. The contact forces acting in such joints are of a particular importance in the design and analysis of spherical joints [5-7]. It was also analyzed, based on contact forces, the energy dissipated in a spherical joint $[8,9]$.

Recent studies have analyzed how the contact forces within the spherical joints that form space structures affect their stability and precision [10-12].

Liu et al. [13] have developed a mathematical model for approximation of the dimensional tolerance of a spherical joint which is based on distributed elastic forces.

\section{Geometrical modeling of the spherical joint}

Let us consider a kinematic chain of a 6 RSS parallel mechanism (Figure 1). The kinematic chain is composed of rotational joints in the points $O_{\mathrm{k}}$ and spherical joints in the points $A_{\mathrm{k}}$ and $B_{\mathrm{k}}$, respectively. The actuating arms $a_{\mathrm{k}}=O_{\mathrm{k}} A_{\mathrm{k}}=254 \mathrm{~mm}$ are rotated at an angle $\theta_{\mathrm{k}}$ around the axis passing through the $O_{\mathrm{k}}$ point, by means of actuators mounted on the base plate. The position of the $O_{\mathrm{k}}$ point relative to the $\{R\}$ system, the length of the actuating arms $k$ and the value of the angle $\theta_{\mathrm{k}}$ are known. In Figure 2, has been shown the position of the $O_{\mathrm{k}}$ points.

\footnotetext{
* Corresponding author: milica.lucian@ugal.ro
} 
Also, it has been noted with $e=106 \mathrm{~mm}$ the apothem of the base and with $g=$ $76 \mathrm{~mm}$ the distance $O_{\mathrm{k}} O_{\mathrm{k}+1}$ which is the same distance as $B_{\mathrm{k}} B_{\mathrm{k}+1}$.

Let's look at one of the six kinematic chains of the 6RSS parallel mechanism (Figure 3). The degree of mobility of the mechanism is given by the Grübler-Kutzbach formula:

$$
M=6 \cdot m-\sum_{n=1}^{5} c \cdot k_{n}=6 \cdot 13-(6 \cdot 5+12 \cdot 3)=12
$$

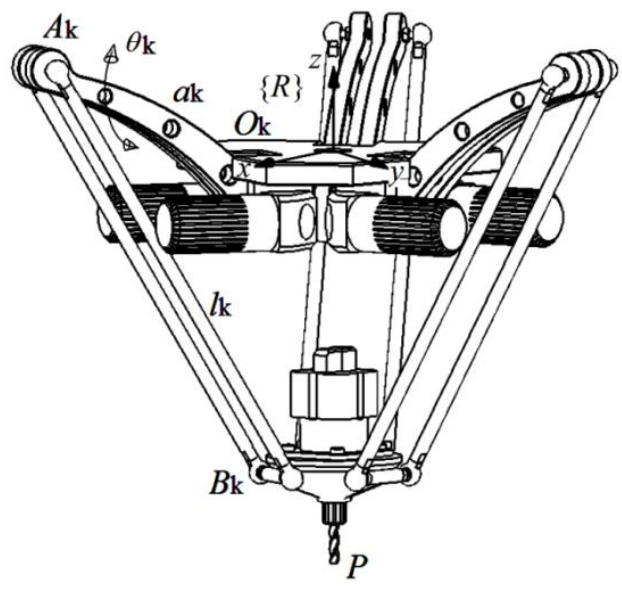

Fig. 1. Highlighting the $O_{\mathrm{k}}, A_{\mathrm{k}}, r_{\mathrm{k}}, \theta_{\mathrm{k}}$ parameters of the new $6 \underline{R} S S$ parallel manipulator.

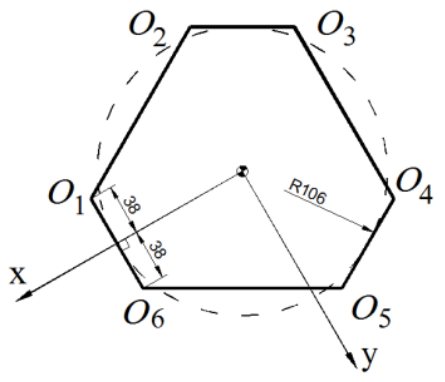

Fig. 2. The position of the $O_{\mathrm{k}}$ centers of the rotational joints.

Each actuating arm has a local mobility given by the rotation around the $A_{\mathrm{k}} B_{\mathrm{k}}$ axis, independent motion relative to the other possible motions of the mechanism. Eliminating these six isolated mobilities it results $M=6$.

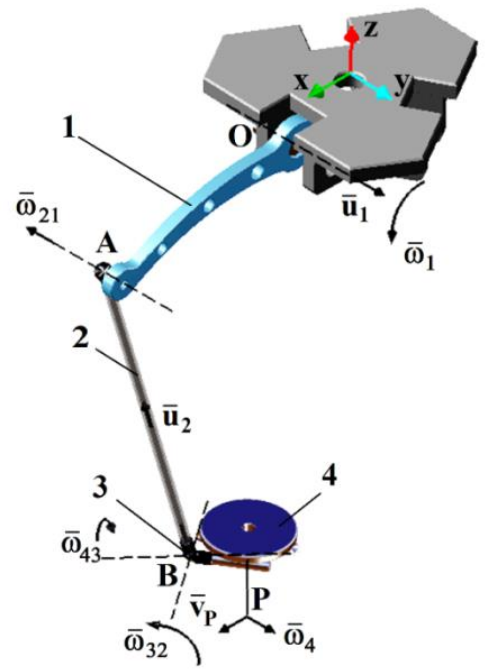

Fig. 3. Representation of a kinematic chain of the mechanism.

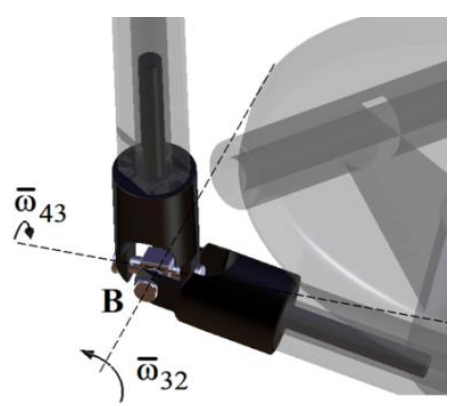

Fig. 4. Highlighting the $4^{\text {th }}$ degree kinematic joint for the $B$ point.

In the analysis of mobility it has been defined the following parameters:

$-m=n+1$ ( $m$ represents the number of mobile kinematic elements);

$-c$ is the class of the kinematic joints;

- $k_{n}$ is the number of kinematic joints of class $n$. 
For a more intuitive representation, it has been replaced the spherical joint from point $B$ with a U-joint. This modification does not affect the degree of mobility of the mechanism. The direct kinematic model involves determining the speed of point $P$, based on six systems of equations, one for each kinematic chain (2):

$$
S=\left\{\begin{array}{l}
\omega_{1} \times O P+\omega_{21} \times A P+\omega_{32} \times B P+\omega_{43} \times B P=V_{P} \\
\omega_{1}+\omega_{21}+\omega_{32}+\omega_{43}=\omega_{4}\left(\omega_{4} \equiv \omega_{P}\right)
\end{array}\right.
$$

The variables in this case are: $\omega_{1}, \omega_{32}, \omega_{43}, \boldsymbol{\omega}_{21}, \boldsymbol{\omega}_{4}, \mathbf{v}_{\mathbf{P}}$.

Using the inverse kinematic model in which is known the linear speed $\left(\mathbf{v}_{\mathbf{P}}\right)$ and the angular speed $\left(\boldsymbol{\omega}_{4} \equiv \boldsymbol{\omega}_{\mathrm{P}}\right)$ of point $P$, it can be determined six scalar variables: $\omega_{1}, \omega_{32}$, $\omega_{43}, \omega_{21}^{x}, \omega_{21}^{y}, \omega_{21}^{z}$.

\section{Application for the inverse kinematic model}

Let's look at the spherical joint of one of the points $A_{\mathrm{k}}$.

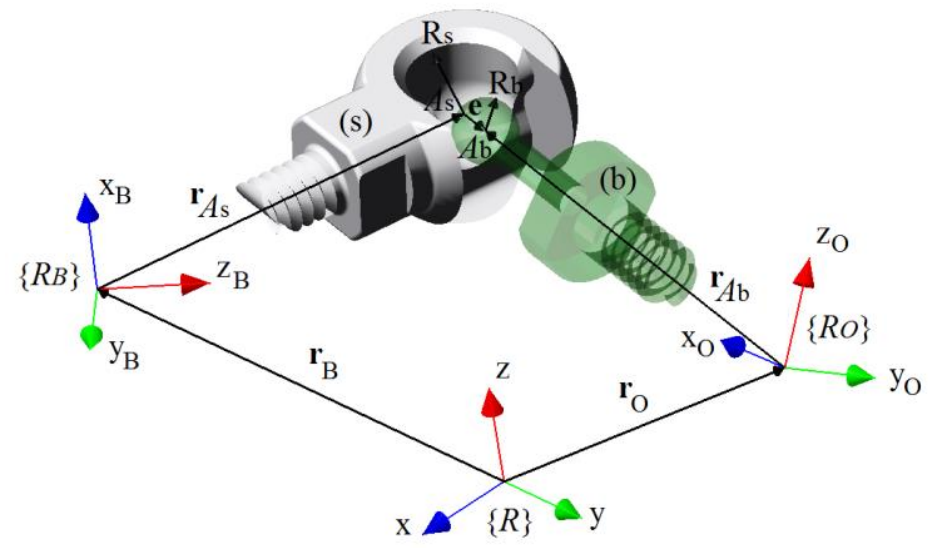

Fig. 5. Representing spherical joint with point position $A_{\mathrm{s}}$ and $A_{\mathrm{b}}$.

Let's consider two bodies $b$ (ball) and $s$ (socket) that represent the spherical part and the concave part of a spherical joint from point $A_{1}$ (Figure 5).

The spherical part of the body $b$ is inside the concave part of the body $s$. It will be noted with $\mathrm{R}_{\mathrm{s}}$ and $\mathrm{R}_{\mathrm{b}}$ the radius of the spherical body $b$ and the concave body $s$. The difference between the radius $\mathrm{R}_{\mathrm{s}}$ and $\mathrm{R}_{\mathrm{b}}$ is the radial tolerance denoted by $j=\mathrm{R}_{\mathrm{s}}-\mathrm{R}_{\mathrm{b}}$ expressed in the above figure by the vector $\mathbf{e}$. The orientation of this vector coincides with the one of the axis passing through the points $A_{\mathrm{s}}$ and $A_{\mathrm{b}}$, identical to the axis of the body $b$.

The position of the point $A_{\mathrm{S}}$ in the system $\left\{R_{B}\right\}$ is given by the position vector $\mathbf{r}_{\mathrm{A}_{\mathrm{s}}}$ and the position of the point $A_{\mathrm{b}}$ in the system $\left\{R_{O}\right\}$ by the position vector $\mathbf{r}_{\mathbf{A}_{\mathbf{b}}}$. Also, $\mathbf{r}_{\mathbf{B}}$ and $\mathbf{r}_{\mathbf{O}}$ are positional vectors of system origin $\left\{R_{B}\right\}$ and $\left\{R_{O}\right\}$ respectively. Norm of the vector $\mathbf{e}$ in the global system $\{R\}$ is:

$$
|\mathbf{e}|=\sqrt{\left(x_{A}^{b}-x_{A}^{s}\right)^{2}+\left(y_{A}^{b}-y_{A}^{s}\right)^{2}+\left(z_{A}^{b}-z_{A}^{s}\right)^{2}}
$$

It has been done an application in which was considered the characteristic point $P$ in motion on a spatial curve of length $\mathrm{L}=528.637 \mathrm{~mm}$, when $\beta \neq \gamma \neq 0=c t$ and $\alpha$ is variable $(\alpha, \beta, \gamma$ are the three orientation parameters of the mobile platform). In this application, the point $P$ is in permanent contact with the curve. It has been stated that in the 
application done through the Catia software, the motion parameter is an arc of curve by equal length, dividing the curve into 72 equally distributed points on it, whose Cartesian coordinates are returned by the program.

The particularity of the application is that the inertia forces $\mathbf{F}_{\mathbf{i}}$ are neglected, the motion being performed very slowly, therefore, there is only one axial force $\mathbf{F}_{\mathbf{2 1}}$ acting in the $\mathbf{A B}$ direction.

Due to the imposed initial condition - the force acts in the $\mathbf{A B}$ direction - the eccentricity $\mathbf{e}$ is manifested in this direction throughout the motion. At the opposite pole of this eccentricity is the point of contact $k$ between the spherical head and the concave part of the spherical joint (Figure 6).

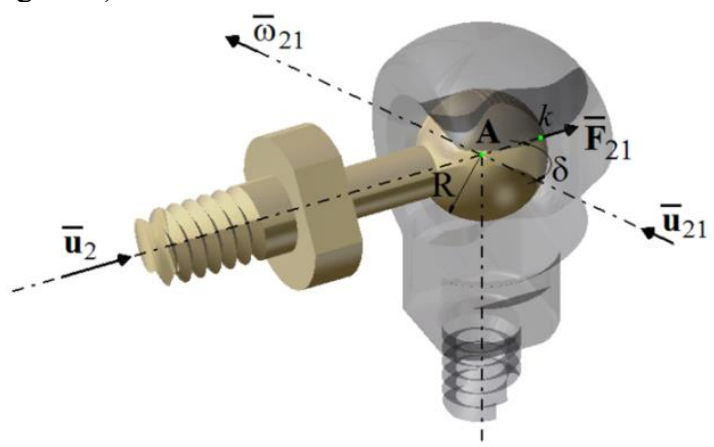

Fig. 6. Highlighting the point of contact $k$ of the spherical joint $A$.

It has been noted with $\delta$ the angle between versor $\mathbf{u}_{2}$ and the versor $\mathbf{u}_{21}$ and with $\mathrm{R}$ the radius of the spherical head of the joint. Also $\boldsymbol{\omega}_{21}$ is the angular velocity of the contact point $k$.

Taking into account the imposed condition $\mathbf{F}_{\mathbf{i}}=0$, it can be written the following expression for the power loss $\mathbf{P}_{\mathbf{f}}$ determinated by friction between the kinematic joint elements in point $A$ :

$$
\mathbf{P}_{\mathbf{f}}=\mu \cdot \mathbf{F}_{21} \cdot \boldsymbol{\omega}_{21} \cdot \mathrm{R} \cdot \sin \delta
$$

The mechanical work of friction forces is:

$$
\mathbf{L}_{\mathbf{f}}=\int_{0}^{\mathrm{T}} \mathbf{P}_{\mathbf{f}} \mathrm{dt}=\mu \cdot \mathbf{F}_{21} \cdot \mathrm{R} \int_{0}^{\mathrm{T}}\left(\boldsymbol{\omega}_{21} \cdot \sin \delta\right) \mathrm{dt}
$$

It can be considered the axial force $\mathbf{F}_{\mathbf{2 1}}$ constant and unitary $\left(\mathbf{F}_{\mathbf{2 1}}=\mathrm{ct}=1\right)$ throughout the entire during movement.

The value of the angle $\delta$ is given by the expression:

$$
\cos \delta=\frac{\mathbf{u}_{2} \cdot \boldsymbol{\omega}_{21}}{\left|\mathbf{u}_{2}\right| \cdot\left|\boldsymbol{\omega}_{21}\right|} \rightarrow \sin \delta=\sqrt{1-\cos ^{2} \delta}
$$

Based on of the Catia software, it can be determined the components of the vector $\boldsymbol{\omega}_{21}$, on the three directions $\left(\omega_{21}^{x}, \omega_{21}^{y}, \omega_{21}^{z}\right)$. Also, it can be determined the components $\left(u_{2}^{x}\right.$, $\left.u_{2}^{y}, u_{2}^{z}\right)$ of the $\mathbf{u}_{2}$ versor of the spherical head axis.

From the six scalar components is resulting (7) and (8):

$$
\begin{aligned}
& \left|\boldsymbol{\omega}_{21}\right|=\sqrt{\omega_{21}^{x^{2}}+\omega_{21}^{y^{2}}+\omega_{21}^{z}{ }^{2}} \\
& \mathbf{u}_{\mathbf{2}} \cdot \boldsymbol{\omega}_{21}=\omega_{21}^{x} \cdot u_{2}^{x}+\omega_{21}^{y} \cdot u_{2}^{y}+\omega_{21}^{z} \cdot u_{2}^{z}
\end{aligned}
$$


The integration of formula (5) was done numerically with the trapeze method, using the finite formula:

$$
\mathbf{L}_{\mathbf{f}} \approx \mu \cdot \mathbf{F}_{\mathbf{2 1}} \cdot \mathrm{R} \sum_{i=1}^{72}\left(\boldsymbol{\omega}_{21} \cdot \sin \delta\right)_{i} \cdot \Delta t
$$

where:

$$
\Delta t=\frac{T}{72} \mathrm{~s}
$$

represents the time required to go through one of the 72 equal circle arcs.

Admitting a constant speed of motion of the point on the curve, $v=10 \mathrm{~mm} / \mathrm{s}$, and knowing its length ( $\mathrm{L}$ ), results the time T required for following the curve:

$$
T=\frac{L}{v}=52.8637 \mathrm{~s} \rightarrow \Delta t=0.7342 \mathrm{~s}
$$

In Figures 7 and 8 it has been represented the variation of vector components $\boldsymbol{\omega}_{21}$ and $\mathbf{u}_{\mathbf{2}}$ depending on the position of the $P_{\mathrm{j}}$ point on the curve.

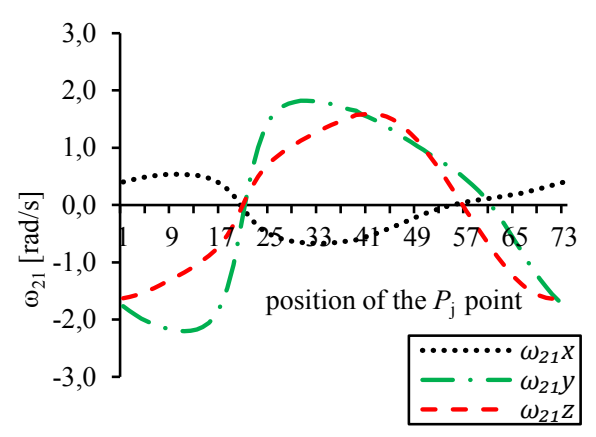

Fig. 7. Vector components $\boldsymbol{\omega}_{\mathbf{2 1}}$ depending on the position of the $P_{\mathrm{j}}$ point.

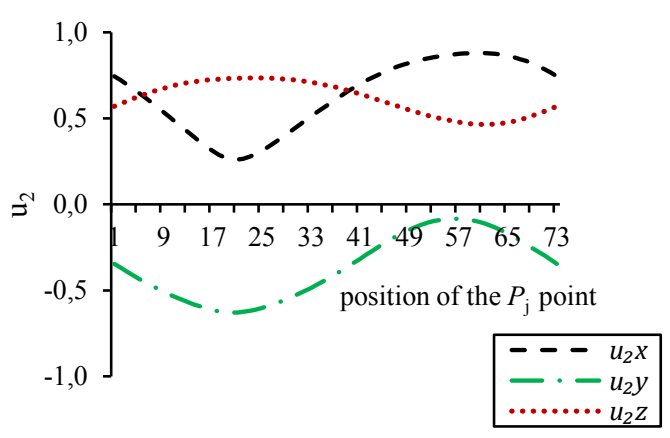

Fig. 8. Vector components $\mathbf{u}_{2}$, depending on the position of the $P_{\mathrm{j}}$ point.

By admitting a friction coefficient $\mu=0.2$, from (4), (6), (7) and (8) it can be determined the lost power by friction $\mathbf{P}_{\mathbf{f}}$ in the kinematic joint of point $A$ for each position $P_{\mathrm{j}}$ of the characteristic point (Figure 9). By summing these values, it has been obtained the frictional power losses in the $A$ kinematic joint, when the characteristic point follows the curve.

$$
\mathbf{P}_{\mathbf{f}}=\mu \cdot \mathrm{R} \sum_{i=1}^{72}\left(\boldsymbol{\omega}_{21} \cdot \sin \delta\right)_{i}=0.13 \mathrm{~W}
$$

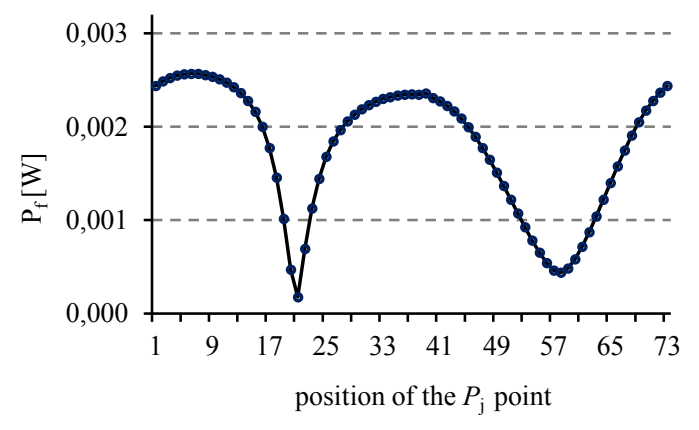

Fig. 9. Frictional power loss $\mathbf{P}_{\mathbf{f}}$, for each position of the $P_{\mathrm{j}}$ characteristic point. 
Also, it can be determined, using the functions of Catia software, the trajectory of a point $k^{\prime}$ belonging to the spherical head of the joint on its concave surface.

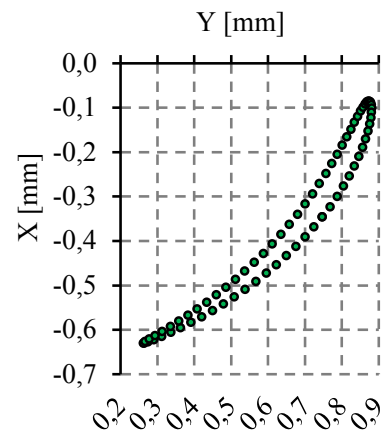

Fig. 10. Projection of the $k^{\prime}$ point trajectory on XY plane.

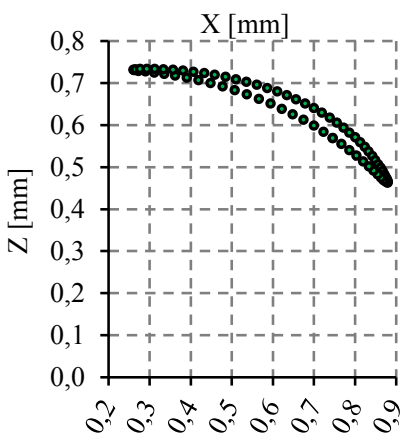

Fig. 11. Projection of the $k^{\prime}$ point trajectory on ZX plane.

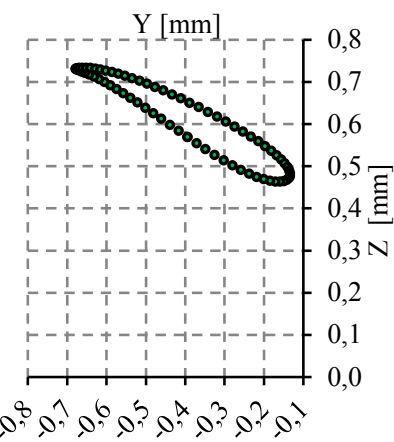

Fig. 12. Projection of the $k^{\prime}$ point trajectory on ZY plane.

Projecting the coordinates of these points on the three plans $\mathrm{XY}, \mathrm{XZ}, \mathrm{ZY}$, it can be obtained the diagrams of Figures 10-12 which describe the trajectory of point $k^{\prime}$.

\section{Conclusions}

This paper presents an original method for determining the power losses due to friction between the components of the spherical joints belonging to a $6 \underline{R S S}$ parallel mechanism.

The determination of these power losses in spherical joints was done in an application where, the characteristic point $P$ follows a closed space curve, neglecting the inertial forces and the motion being very slow.

Using the Catia software, the variation graphs of the versor $\mathbf{u}_{2}$ of the direction $\mathbf{A B}$ and of the vector $\boldsymbol{\omega}_{21}$ have been determined depending on the position of the point $P_{\mathrm{j}}$ on the given spatial curve. The trajectory of a point belonging to the spherical head of the spherical kinematic joint of the parallel mechanism was obtained and the graphs of the traiectory's projections on the three plans XY, ZX, YZ were done.

\section{References}

1. S. Dubowsky, F. Freudenstein, ASME J. Eng. Ind., 93, 305-309 (1971)

2. P. Flores, J. Ambrósio, J. P. Claro, H. M. Lankarani, C.S. Koshy, Mech. Mach. Theory, 41, 247-261 (2006)

3. O.A. Bauchau, J. Rodriguez, Int. J. Solids Struct., 39, 41-63 (2002)

4. P. Flores, Nonlinear Dyn., 61, 633-653 (2010)

5. F. Farahanchi, S. W. Shaw, J. Sound Vib., 177, 307-324 (1994)

6. G. S. Boltachev, N. B. Volkov and N. M. Zubarev, Int. J. Solids Struct., 49, 21072114 (2012)

7. J. Rhee, A. Akay, Mech. Mach. Theory, 31, 121-134 (1996)

8. P. Flores, J. Ambrósio, and J. P. Claro, Multibody Syst. Dyn., 12, 47-74 (2004)

9. M. Lankarani, and P. E. Nikravesh, ASME J. Mech. Des., 112, 369-376 (1990)

10. J. A. C. Ambrósio, NATO Adv. Sci. Inst., 2, 15-33 (2002)

11. M. A. Brown, Adv. Space Res., 48, 1747-1753 (2011)

12. Z. H. Qi, Y. S. Xu, X. M. Luo, and S. J. Yao, Multibody Syst. Dyn., 24, 133-166 (2010)

13. C. S. Liu, K. Zhang, and L. Yang, J. Comput. Nonlin. Dyn., 1, 160-167 (2006) 